Research Article

Yalcin Yildirim* and Mahyar Arefi

\title{
Noise complaints during a pandemic: A longitudinal analysis
}

https://doi.org/10.1515/noise-2021-0008

Received Dec 14, 2020; accepted Jan 28, 2021

\begin{abstract}
COVID-19 has affected people's lives in different ways from reduced mobility and staying-at-home orders to other daily life routines. These changes have, in turn, affected the quality of life in urban environments including air quality and noise. The noise aspect, for example, suggests quieter environments due to fewer vehicles on streets, and less human activities. On the other hand, staying at home may cause more activities happening at the building level, i.e., more people in buildings may make more noise for neighbors. In order to understand this nexus, the study examines the noise complaints data in Dallas, USA. To do this, the study first compares the noise complaints after the COVID-19 intercourse and the same data period in 2019. Findings surprisingly show reduced noise complaints during the COVID-19 time frame by about 14\% compared to the pre-COVID-19 period. The majority of this reduction occurred in and around the city center. In other words, the noise complaints seem more spatially dispersed at the outskirts of the city. Another finding that directs more detailed analyses, however, considers the massive reduction of ridership, traffic circulation, and building permits. This needs some other techniques for determining the sources for incommensurate noise complaints.
\end{abstract}

Keywords: COVID-19, noise complaints, 311 data, city center, suburbs

\section{Introduction}

Once officially identified, the first COVID-19 case in China rapidly spread all over the world. With the first case reported on January $20^{\text {th }}$, the government declared a public health emergency at the end of January 2020. An immediate application of a number of international restrictions and

\footnotetext{
`Corresponding Author: Yalcin Yildirim: Bursa Technical University, Department of Landscape Architecture, Bursa, 16310, Turkey; Email: yalcin.yildirim@btu.edu.tr

Mahyar Arefi: Jundi Shapur Technical University, Dezful, Iran
}

controls ensued national-level actions, including testing and travel restrictions. After announcing the strict COVID Act in March 2020, though the U.S. did not mandate lockdown, regional and local officials considered several reactions, i.e., canceling large gatherings, stay at home orders, and school closures.

Fewer commutes as well as reduced outdoor human activities and witnessing new urban lifestyles, travel behaviors, and home-bound adaptations were associated with other observations such as the cleaner air quality, and noise reduction in the built environment. Staying at home allows people to have more time for various activities such as watching TV, listening to' loud music, workout activities, home repairing, kids' play, etc. Thus, while trafficrelated and other outdoor anthropogenic activities may reduce noise complaints, staying at home may increase other forms of noise. Consequently, the prolonged COVID-19 era has stimulated noise complaints for various reasons. In order to understand the direct linkage between COVID-19 and noise complaint requests, this study has culled and analyzed them from March through December 2020, and has compared the same sourced noise complaints with the same period last year.

\section{Related studies}

\subsection{COVID-19 and Noise}

Several studies have examined the relationship between the unpredicted COVID-19 outbreak and noise from various aspects. Perhaps as a pioneer study in examining noise during the COVID-19, Aletta et al. created a traffic simulation that represented noise pollution in Rome, Italy [1]. Their findings report almost $65 \%$ reduction in both traffic and noise emissions during the lockdown [1]. Pagès et al.’s [2] study in the same location and Milan examined the effects of the COVID-19 on traffic and non-traffic noise activities by using the DYNAMAP system. To do this, the authors compared the same noise events in the same period of time in 2019. Aletta et al. [3] collected soundscape assessments from 11 locations in London before the 2019 lockdown, and 
during the 2020 lockdown. The study shows a $5.4 \mathrm{~dB}\left(\mathrm{LA}_{e q}\right)$ noise reduction within the author-based urban setting classifications during the lockdown period. Sakagami reported results in a study that shows a small difference between the sound measurements in June 2020, and the previous year [4]. The author triggers two interpretations based on the findings that either the noise levels were lower during the state of emergency or excessively higher immediately after the cancellation of the state of emergency. De Laura et al. also examined the soundscape at a relatively smaller scale location (Fontana di Trevi- in Rome, Italy), where they collected sound samples [5]. Their findings help differentiate the anthropogenic effects of sound levels. Basu et al. examined noise levels in Dublin, Ireland by comparing preand post-lockdown periods from January to May 2020, and found pre-lockdown noise levels to be higher than the ones collected during the lockdown [6]. In another study, Rumpler et al. investigated the noise level variations during the COVID-19 period in Stockholm, Sweden [7]. To show the variation, the authors account for the restriction days in April 2020 and found noise levels considerably lower during the measurement period [7]. Čurovič et al. assessed the noise level variance at the port vicinity by comparing preCOVID-19, and during the COVID-19 period, measured the noise levels in three locations, and found that evening and night measurements showed around $25 \%$ reductions during the COVID-19 period [8]. Mostafa et al. conducted a comprehensive environment-related investigation including air pollution indicators, solid wastes, and noise in Egypt, and reported a strong negative link, specifically $75 \%$ noise measurement reduction [9]. Covering various regions in the world in regards to the nexus between COVID-19 and noise shows a literature gap in North America, particularly the U.S. In only one study [10], volunteer participants used their Apple watch and headphone sound-related data in four states. The authors collected 8-hour normalized exposure sound data for both COVID-19 initial date and intervention date with 5,894 participants. Their findings show an approximately $3 \mathrm{dBA}$ noise level reduction, therefore claiming that everyday life had less exposure to noise due to the COVID-19 restrictions. However, in addition to collecting sound parameter-related studies, residents who actually experience noise in their daily life could report potential changes and their relative consequences. Noise complaint records show one way of acting this way.

\subsection{System and noise complaints}

Noise complaints in the U.S. partly reflect 311 nonemergency city services including self-reported concerns, resident requests, and occasionally visitors as service standards for urban governance [11]. Records for this service offer people to report concerns that reflect the quality-oflife aspects of daily lives [11]. Several studies have used 311 system data to identify urban environment-related circumstances. Wang et al. represent this dataset by examining New York City, Boston, and Chicago cases where they clustered various 311 data-related urban context patterns and concluded that such efforts may help both refine local city solutions, and clarifying resident concerns [11]. Other studies also covered the 311 system analytics, including smart city dynamics [12], socio-spatial disparity patterns [13], public health examination of discarded needles [14], and urban informatics of the planning field [15].

As with noise specification, few studies assessed the 311 data or similar systems. Minkoff investigated the 311 system-related engagement for the census-tract level data on government-provided goods, graffiti, and noise in New York City [16]. They showed how noise complaints reflect resident behavior or built-environment features that cause annoying environmental noise [16]. In a pioneer study examining the nexus between noise and 311 datasets, Duncan et al. assessed noise and socio-demographic aspects of neighborhood features in New York City, and found a significant difference in neighborhood noise complaints, particularly in low-income areas [17]. Tariq et al. performed machine learning algorithms such as Decision Tree, Random Forest, Naive Bayes, etc., by using noise data of the 311 datasets [18]. The study shows that Convolutional Neural Networks (CNN) performs best with such data compared to other models [18]. Liu et al. examined the entire gamut of resident complaints in Brisbane, Australia, and performed a number of analyses to find patterns between four-classified complaints: animal-related, building construction, property management, and health [19]. The study examined the following noise categories: animals, builders' and other sources [19]. To understand the relationship with other factors, another study examined data on 311 noise complaints in Vancouver, Canada [20]. The findings show a strong relationship between noise complaints and construction activities [20].

While the studies discussed in the former section mainly assessed noise level measurements, other methods account for noise concerns including residents' self-reports. While somewhat similar, this study examines the nexus between COVID-19 and noise from a different angle. Arguably, no study has investigated such a relationship based on self-reported data. This study gathered the residents' selfreported noise complaints data after the COVID-19 outbreak in Dallas, Texas, and compared them with the same period in 2019. This study seeks two key answers within its scope: 
1) Have people called 311 city service to report more noiserelated complaints, or not during the COVID-19? 2) Does a spatial pattern of noise complaints exist between before and during the COVID-19 periods?

\section{Materials and methods}

\subsection{Study area}

With over 1.2 million population, as the third-largest city in the state of Texas, and the ninth in the U.S., Dallas provides an appropriate case study for noise-related concerns. Wellpositioned in the core of the Dallas-Fort Worth Metropolitan Statistical Area with approximately 7 million people, and located 245 miles north and northwest of Houston, and 300 miles north of the Gulf of Mexico, Dallas attracts many businesses 20 of which rank within the Fortune 500 companies. With these characteristics and like many other large cities, Dallas has experienced both emergency and nonemergency situations.

\subsection{Materials}

The data used in this study came from Dallas, and comprise calls and requests from 311 about residents' communities [21]. The requests and complaints range from home, traffic circulation, traffic signals, parking, sidewalks, public health, safety, and noise. The data include details including addresses, service request numbers, service request types, city council numbers, dates-related information, priority types, methods of report, longitude and latitude information, and so on. To achieve appropriate formats for understanding the on March 2019, and to conduct comparable assessments, the authors applied various filtering and clearing processes. As many other studies have pointed out, the 311 noise data determine trouble and pollution indicators that come from Dallas residents $[17,22]$.

As a methodological procedure, the research mainly aimed to compare the previous and during the COVID-19 period data both spatially and statistically. To observe differences, the study first compared monthly noise complaints, followed by a spatial examination of detecting potential before and during COVID-19 periods patterns. As a unique aspect of this method, the study adopted small scale spatial units. To examine the nexus between noise complaints and distance from the city center that also covered multiple buffer zone proximities, it also set up a particular procedure. Lastly, to ensure spatial understanding, a two-way ANOVA statistical analysis factored in the distance from the city center, and the time period for reporting noise complaints.

\section{Findings and results}

After performing thereof analyses and mapping procedures, several findings emerged. The research examined a total of 4315 noise complaints from March to December 2019, and March to December 2020. Of these, 2315 complaints were reported in the former period, and 2000 complaints in the latter. In other words, the noise complaints show $13.6 \%$ reduction after the COVID-19 breakout period compared to the same period in 2019. To identify the micro-details with a different lens, the study also examined these cases month by month. As shown in figure 1, overall, each month in 2020 includes lower noise complaints at about $4 \%$ to $40 \%$, except for September that reports $20 \%$ more noise complaints compared to 2019. The lowest noise complaints occurred in November, July, and August during the COVID2019 period, while for 2019, they occurred in July, August, and September, respectively. The highest noise complaints also differ in May and September for the COVID-19 period, and in May and October of 2019. Furthermore, performing the t-test shows no statistical difference between the months $\left(t_{(16)}=1.893, p=0.077\right)$.

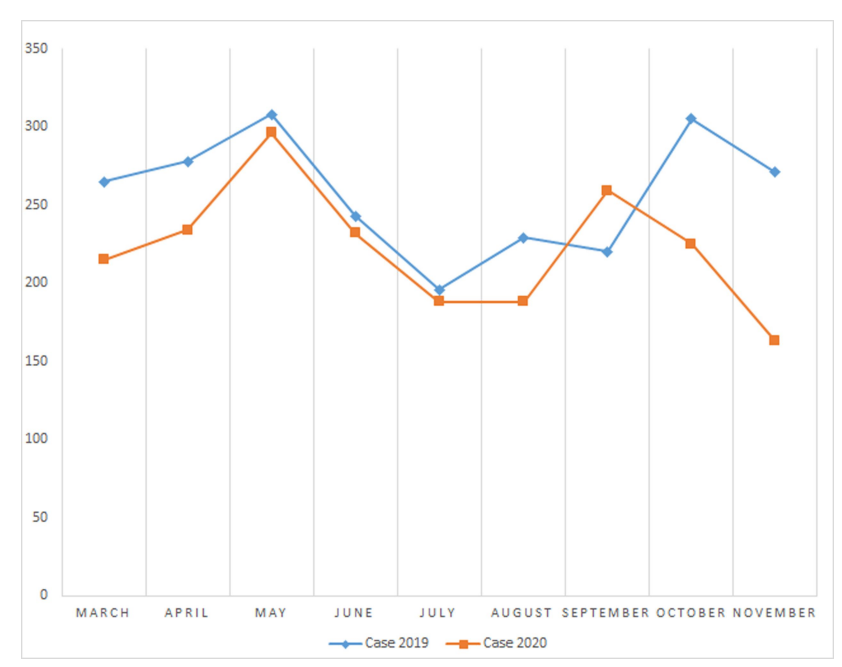

Figure 1: Monthly noise complaints comparison of before the COVID19 and during the COVID-19

To better understand, the study also examined the noise complaints data spatially and generated maps using the ArcGIS Tools (10.7.1 version). As the first noticeable difference, the spatial maps (Figure 2), show the re- 


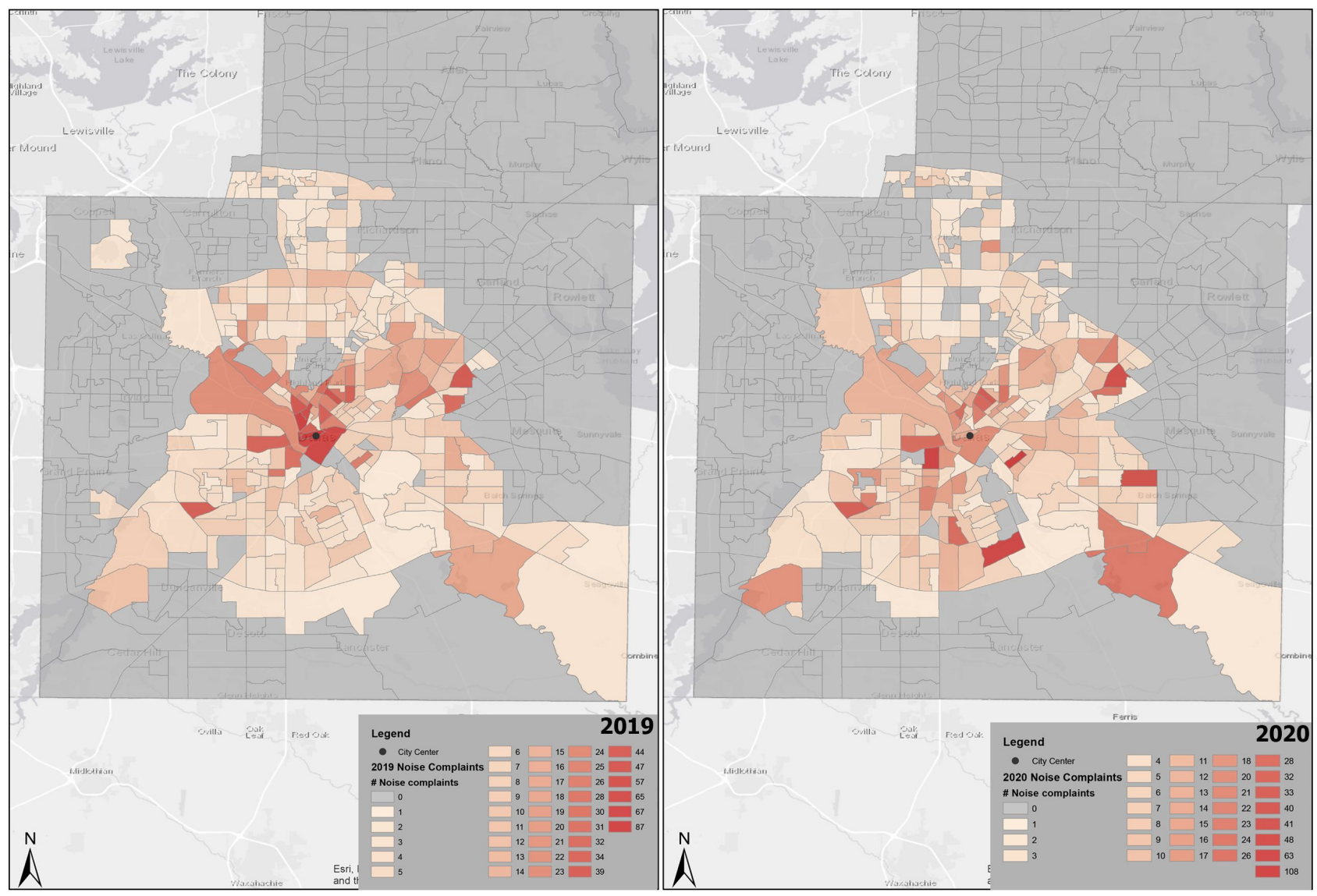

Figure 2: Noise complaints comparisons of before the COVID-19 and during the COVID-19 at the census tract level

Table 1: Number of noise complaints and highest census tracts of buffer zones

\begin{tabular}{lccccc}
\hline Category & $\mathbf{1}$ mile & $\mathbf{3}$ mile & $\mathbf{5}$ mile & $\mathbf{8}$ mile & $\mathbf{+ 8}$ mile \\
\hline Before COVID-19 & $186(3)$ & $439(10)$ & $138(4)$ & $205(6)$ & $111(2)$ \\
During COVID-19 & $45(0)$ & $379(4)$ & $117(5)$ & $241(9)$ & $176(7)$ \\
No Change & $0(0)$ & $13(2)$ & $14(4)$ & $39(11)$ & $32(13)$ \\
\hline
\end{tabular}

ported noise complaints before the COVID-19 period clustered more around the city center, while during the COVID19, the complaints show a more dispersed pattern surrounding the city. Furthermore, pre-COVID-19 noise complaints show higher concentrations in north and northwest directions compared with south and southwest directions during the COVID-19 period.

This evidence-based pattern required further detailed spatial examinations. The study filtered the census tracts that include more noise complaints compared to another year as well as whether those that present no change in terms of the number of noise complaints. To demonstrate this, Figure 3 shows the noise complaints' spatial variance, where the census tracts with the yellow color include more noise complaints during the COVID-19 compared to the pre- vious year (the highest 25 census tracts). The census tracts with blue show more noise complaints in 2019 compared to the COVID-19 breakout period (the highest 25 census tracts). Finally, the census tracts with white color represent the same number of noise complaints (not zero) both before the COVID-19 and during the COVID-19 (Total 30 census tracts).

The study also examined possible spatial patterns or lack thereof in noise complaints. Establishing buffer zones from the city center serves as a method of observing the noise complaints that allow researchers to understand the city center effects. To do this, five categories of buffer zones - 1 mile, 3 miles, 5 miles, 8 miles, and +8 miles - helped analyze the data for a meaningful interpretation. As Table 1 illustrates, the pre-and during COVID-19 census tracts show 
no change between these periods (except 0 value) in the five buffer zones. While the numbers in parentheses refer to the census tracts with higher noise complaints in certain buffer zones, the numbers represent the quantity of the actual noise complaints. For instance, 186 actual noise complaints were reported in the 3 highest number census tracts before COVID-19, and 45 noise complaints during COVID-19 with 0 highest number census tracts, and not any "no change"

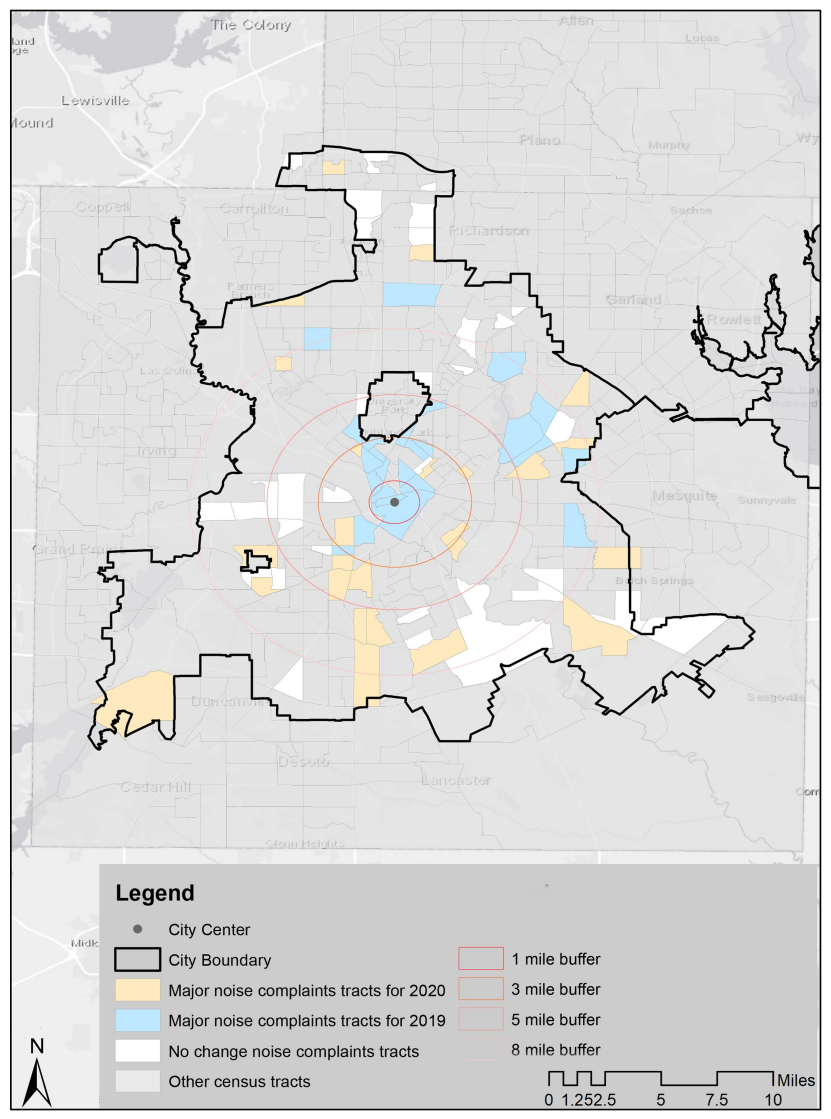

Figure 3: Census tracts with major noise complaints before the COVID-19 and during the COVID-19 noise complaints in census tracts within 1-mile buffer zone of the city center. The rest of the table can be interpreted with similar instructions.

The study also examined "trade-offs" for changing patterns on noise complaints before and during COVID-19 (in case they existed). As Figure 4 shows, the left figure represents the number of noise complaints and their change with distance while the figure on the right refers to the number of highest representative census tracts and their change with distance from the city center. Surprisingly, both the number of noise complaints and the highest census tracts show similar patterns with an increasing trend, where before COVID-19 cases are higher than during the COVID-19 cases for 1-mile and 3-mile distance from the city center. Both categories decrease with the same pattern at about a 5 -mile buffer zone. Then, the odds change in a way that the number of noise complaints increases in the highest census tracts during rather than pre-COVID-19 conditions. After a 5-mile distance, the gap between proximity and number of cases gets larger in the same pattern as during COVID-19. This pattern demonstrates an interesting spatial aspect of noise complaints that while showing more concentration in the city center before the COVID-19 period time, they get surprisingly dispersed around the outskirts of the city - more specifically around a 5-mile distance, during the COVID-19.

The two-way ANOVA test results also confirm the pattern that represents the main effects including both the distance from the city center and the comparison period of time in the census tracts have $p=0.000$, though the interaction between distance and time comparisons could not be demonstrated, $F(6,149)=.79, p=0.57$. Last but not least, as Table 2 shows, based on the same test results, adjusted $R$ squared implies that $35.1 \%$ of the variance in the number of noise level complaints belong to proximity to the city center and the period of time measured.

Table 2: Two-way ANOVA Results

\begin{tabular}{lrrrrrr}
\hline Source & $\begin{array}{r}\text { Type III Sum of } \\
\text { Squares }\end{array}$ & df & Mean Square & F & Sig. & $\begin{array}{r}\text { Partial Eta } \\
\text { Squared }\end{array}$ \\
\hline Corrected model & $16826.149^{a}$ & 12 & 1402.179 & 8.259 & .000 & .399 \\
Intercept & 23963.769 & 1 & 23963.769 & 141.156 & .000 & .486 \\
Distance to city center & 5054.343 & 4 & 1263.586 & 7.443 & .000 & .167 \\
Period time & 3785.132 & 2 & 1892.566 & 11.148 & .000 & .130 \\
Distance to city center * Period time & 812.600 & 6 & 135.433 & .798 & .573 & .031 \\
Error & 25295.462 & 149 & 169.768 & & & \\
Total & 67735.000 & 162 & & & & \\
Corrected Total & 42121.611 & 161 & & & & \\
\hline
\end{tabular}

${ }^{a}$ R squared $=.399$ (adjusted R squared $=.351$ ) 

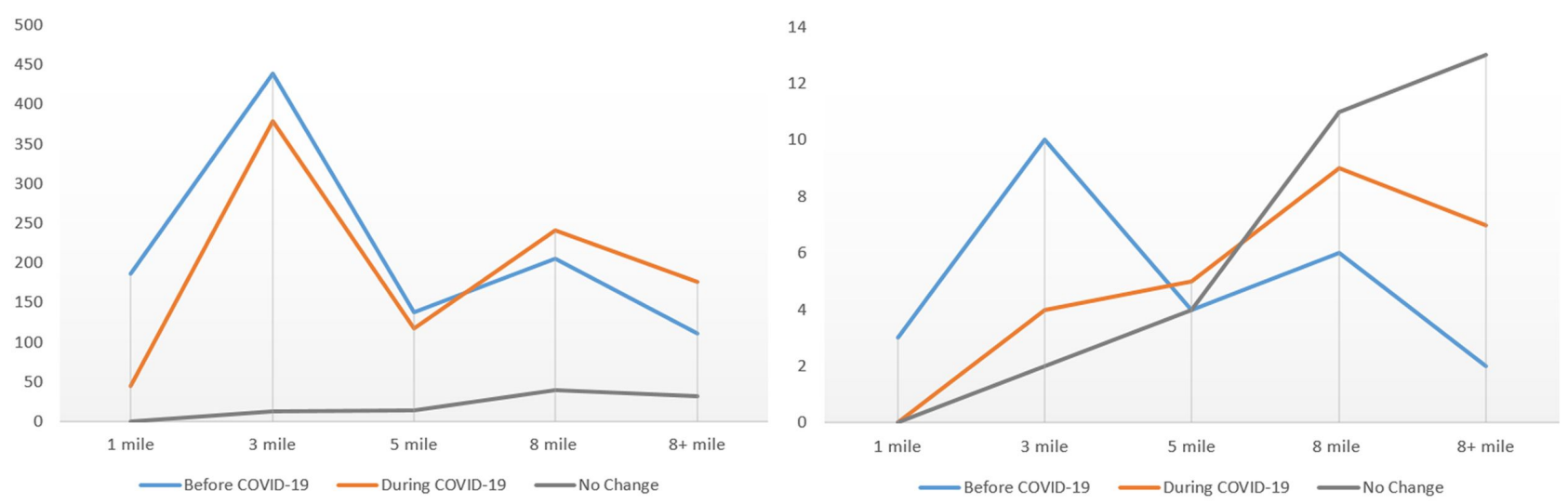

Figure 4: Number of noise complaints and majority census tracts comparisons with the proximity of city center

Table 3: Word frequency of noise complaints in each buffer zone

\begin{tabular}{lllll}
\hline Category & 1 mile & $\mathbf{3}$ mile & $\mathbf{5}$ mile & $\mathbf{8}$ mile \\
\hline & Construction & Noise & Construction & Dumpster \\
& Work & Night & Until night & Weekends \\
Before COVID-19 & Loud & Saturday & Working & Sleep-time \\
& Music & Loud & Loud & Construction \\
& Night & Building & Music & Management \\
\hline & Apartment & Apartment & Neighbor & House \\
& Neighbor & Street & Screaming & Parties \\
During COVID-19 & Music & Night & Bass & Constant music \\
& Street & Parking Lot & Unit & Car \\
& Loud & Music & Ongoing & Days and nights \\
\hline
\end{tabular}

The plot profile of two-way ANOVA illustrates that the estimated marginal means of noise complaints before and during COVID-19 shrinks gradually moving towards out of the city center, though noise complaints during the COVID19 seem to increase compared to before COVID-19, at an about 3-mile distance to the city center. As Figure 5 shows, this pattern continues for about a 5 -mile radius. As spatial analyses also show, the pattern has changed from a 5-mile distance in a way that estimated marginal means during COVID-19 noise complaints increase and continues until the city morphs into suburbs while the before COVID-19 noise complaints reduce gradually with the distance from the city center.

After confirming the statistical and spatial attributes of the noise complaints before and during the COVID-19, the study compared them one more time to obtain a better understanding. Hence, to interpret the most frequently-used words and statements, the noise complaints were coded accordingly. As Table 3 shows, the overarching finding from this examination suggests that the residents' reports of their noise complaints based on the frequency of words vary be-

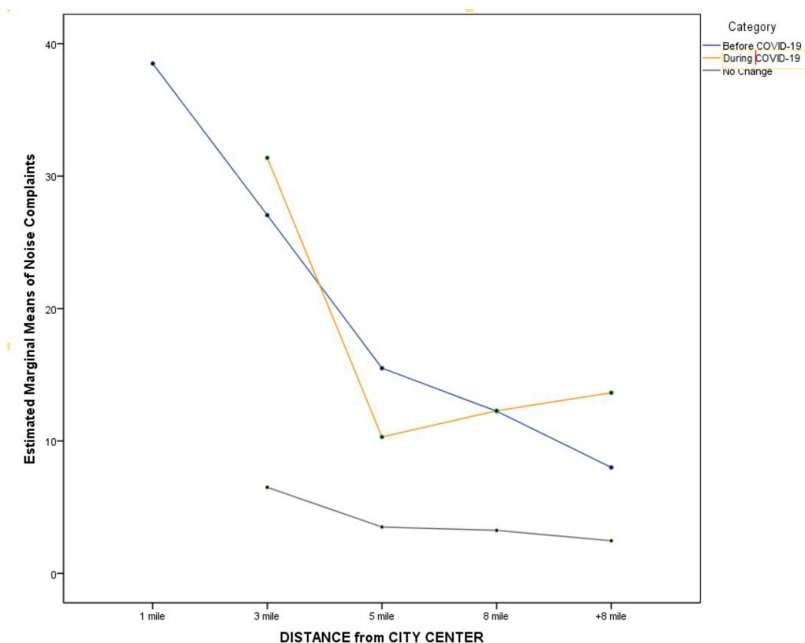

Figure 5: Estimated marginal means and distance from city center among period times

tween the before and during the COVID-19 periods. In most frequently-used word statements in a 1-mile buffer zone, while noise complaints include more "construction, work, 
and night" words before the COVID-19 period, they include "apartment, neighbor, and street" during the COVID-19. In a similar and unique pattern, the 3-mile buffer includes more "apartment, parking lot, music, and street" words during the COVID-19 whilst "noise, night, loud, and Saturday" words were most frequently used before the COVID-19. The difference in using words become more notable with the 5mile buffer that highlights "neighbor, screaming, bass, and unit" words during the COVID-19 and "construction, working, loud, and music" words before the COVID-19. Some other new words emerged in 8-mile buffers and residents used "house, parties, constant music, and car" more during the COVID-19 while "dumpster, weekends, sleep-time, and management” before COVID-19.

\section{Discussion and conclusion}

Using noise complaints data, this study for the first time attempts to examine and conduct different analytical spatial methods by comparing the before and during the COVID-19 time frames.

A total of 4315 noise complaint records accounted for this study show fewer reports during the COVID-19 compared to the same period of time in the previous year. Delving deeper into monthly examination also showed that almost every month, except September in pre-COVID-19 periods included higher noise complaints. Also, analyzing of the most frequently-used words enhances the idea of transforming noise traced back to its sources. So, reduced traffic, ridership, construction, as well as working from home played crucial roles in forming these patterns. Perhaps a more provocative finding shows that the city center has received fewer noise complaints during COVID-19 compared to before the COVID-19 period. Both the spatial and statistical analyses confirm this finding, which goes somewhat contradictory to other studies, i.e., Basu et al. finding that shows less noise reduction in the city center. However, a couple of reasons could explain this difference. First, the studies occurred in completely different regions. Also, while they measured the noise level in various locations in Dublin, this research examined the self-reported noise complaints.

As its limitation, this study did not conduct any noise sampling and only assessed the noise complaints data. However, perhaps future study directions, both in-situ noise sampling and noise complaints could incorporate both of these aspects, thereby, providing a more comprehensive understanding. Also, this study did not include any of the built-environment effects as it could have required a broader examination.

On the other hand, as for the greater reduction of the ridership, traffic movements, construction activities in the city, noise reduction seems relatively lower for the aforementioned dynamics. Hence, to some extent, besides observing a more dispersed noise complaints pattern during the COVID-19 period, these areas could experience some other bothersome noise causes. This situation may require further analyses as stay at home and related home-based activities including children's welfare, home repairs, homerelated hobbies and sports, music and movie sounds, etc. that may generate more noise. This might suggest "relocating modified noise concerns" from city centers to more suburbs needing further studies to confirm such pattern changes in the face of a global pandemic. This also directs further understanding of as some other studies [23, 24] claimed that COVID-19 related noise reduction may result in a decrease in cardiovascular diseases (CVD). Therefore, if our premise in relocating noise concerns through suburbs has some legs, then such noise-related health problems also travel through suburban areas.

Funding information: The authors state no funding involved.

Author contributions: All authors have accepted responsibility for the entire content of this manuscript and approved its submission.

Conflict of Interests: The authors state no conflict of interest.

\section{References}

[1] Aletta F, Brinchi S, Carrese S, Gemma A, Guattari C, Mannini L, et al. Analysing urban traffic volumes and mapping noise emissions in Rome (Italy) in the context of containment measures for the COVID-19 disease. Noise Mapp. 2020;7(1):114-22.

[2] Pagès RM, Alías F, Bellucci P, Cartolano PP, Coppa I, Peruzzi L, et al. Noise at the time of COVID 19: the impact in some areas in Rome and Milan, Italy. Noise Mapp. 2020;7(1):248-64.

[3] Aletta F, Oberman T, Mitchell A, Tong H, Kang J. Assessing the changing urban sound environment during the COVID-19 lockdown period using short-term acoustic measurements. Noise Mapp. 2020;7(1):123-34.

[4] Sakagami K. A note on the acoustic environment in a usually quiet residential area after the 'state of emergency' declaration due to COVID-19 pandemic in Japan was lifted: supplementary survey results in post-emergency situations. Noise Mapp. 2020;7(1):1928. 
[5] De Lauro E, Falanga M, Lalli LT. The soundscape of the Trevi fountain in Covid-19 silence. Noise Mapp. 2020;7(1):212-22.

[6] Basu B, Murphy E, Molter A, Basu AS, Sannigrahi S, Belmonte $M$, et al. Investigating changes in noise pollution due to the COVID-19 lockdown: the case of Dublin, Ireland. Sustain Cities Soc. 2021;65:102597.

[7] Rumpler R, Venkataraman S, Göransson P. An observation of the impact of CoViD-19 recommendation measures monitored through urban noise levels in central Stockholm, Sweden. Sustain Cities Soc. 2020 Dec;63:102469.

[8] Čurovič L, Jeram S, Murovec J, et al. Impact of COVID-19 on environmental noise emitted from the port. Sci Total Environ. 2020;756: 144147.

[9] Mostafa MK, Gamal G, Wafiq A. The impact of COVID 19 on air pollution levels and other environmental indicators - A case study of Egypt. J Environ Manage. 2021 Jan;277:111496.

[10] Smith LM, Wang L, Mazur K, Carchia M, DePalma G, Azimi R, et al. Impacts of COVID-19-related social distancing measures on personal environmental sound exposures. Environ Res Lett. 2020;15(10):104094.

[11] Wang L, Qian C, Kats P, Kontokosta C, Sobolevsky S. Structure of 311 service requests as a signature of urban location. PLoS One. 2017 Oct;12(10):e0186314.

[12] Wu W. Determinants of citizen-generated data in a smart city: analysis of 311 system user behavior. Sustain Cities Soc. 2020;59:102167.

[13] Kontokosta CE, Hong B. Bias in smart city governance: how sociospatial disparities in 311 complaint behavior impact the fairness of data-driven decisions. Sustain Cities Soc. 2020;64:102503.

[14] Bearnot B, Pearson JF, Rodriguez JA. Using Publicly Available Data to Understand the Opioid Overdose Epidemic: Geospatial Distribution of Discarded Needles in Boston, Massachusetts. Am J Public Health. 2018 Oct;108(10):1355-7.
[15] Kontokosta CE. Urban Informatics in the Science and Practice of Planning. J Plann Educ Res. 2018:1-14.

[16] Minkoff SL. NYC 311: A Tract-Level Analysis of CitizenGovernment Contacting in New York City. Urban Aff Rev. 2016;52(2):211-46.

[17] Duncan DT, Tamura K, Regan SD, Athens J, Elbel B, Meline J, et al. Quantifying spatial misclassification in exposure to noise complaints among low-income housing residents across New York City neighborhoods: a Global Positioning System (GPS) study. Ann Epidemiol. 2017 Jan;27(1):67-75.

[18] Tariq A, Shah SK, Lee Y. Smart. 311 Request System with Automatic Noise Detection for Safe Neighborhood, 2018 IEEE Int Smart Cities Conf (ISC2). 2018:1-8.

[19] Liu Y, Cheshire L, Wang S, Fu X. A socio-spatial analysis of neighbour complaints using large-scale administrative data: the case in Brisbane, Australia. Cities. 2019;90:168-80.

[20] Hong A, Kim B, Widener M. Noise and the city: leveraging crowdsourced big data to examine the spatio-temporal relationship between urban development and noise annoyance. Environ Plan B Urban Anal City Sci. 2020;47(7):1201-18.

[21] City of Dallas, Open Data. 311 Service Requests from March 2019 to present.

[22] Salamon J, Jacoby C, Bello JP. A dataset and taxonomy for urban sound research. In: Proceedings of the ACM International Conference on Multimedia, Orlando, Florida, USA: 2017.

[23] Antza C, Stabouli S. Reduction in environmental noise during COVID-19 pandemic and cardiovascular disease: A mystery for further investigation. J Clin Hypertens (Greenwich). 2020 Oct;22(10):1947-8.

[24] Dutheil F, Baker JS, Navel V. COVID-19 and cardiovascular risk: flying toward a silent world? J Clin Hypertens (Greenwich). 2020 Oct;22(10):1945-6. 